\title{
Analysis of the epidemiological and clinical characteristics of 65 patients with scrub typhus on the east coast of China
}

\author{
Pan $\mathrm{Xu}^{1,2 \#}$, Guomin Mao ${ }^{2 \#}$, Haiyan Jiang ${ }^{2 *}$, Yuting Ren², Yue Wang ${ }^{2}$, Guiwen Liang ${ }^{2}$, Wei Liu ${ }^{3}$, \\ Yang Zhou ${ }^{3}$, Zhongwei Huang ${ }^{2}$, Bin Zhang ${ }^{4}$, Xiaodong Chen ${ }^{1}$, Lei Qi ${ }^{2}$ \\ ${ }^{1}$ Department of Dermatology, Affiliated Hospital of Nantong University, Nantong, China; ${ }^{2}$ Department of Emergency Medicine, Affiliated Hospital \\ of Nantong University, Nantong, China; ${ }^{3}$ Department of Obstetrics and Gynecology, Affiliated Hospital of Nantong University, Nantong, China; \\ ${ }^{4}$ Department of Infectious Disease, Affiliated Hospital of Nantong University, Nantong, China \\ Contributions: (I) Conception and design: X Chen, H Jiang, L Qi; (II) Administrative support: L Qi; (III) Provision of study materials or patients: P \\ $\mathrm{Xu}, \mathrm{W}$ Liu; (IV) Collection and assembly of data: G Mao; (V) Data analysis and interpretation: Y Ren; (VI) Manuscript writing: All authors; (VII) \\ Final approval of manuscript: All authors. \\ \#These authors contributed equally to this work. \\ Correspondence to: Lei Qi; Xiaodong Chen; Bin Zhang. Department of Emergency Medicine, Affiliated Hospital of Nantong University, 20 West \\ Temple Road, Nantong 226001, China. Email: qilei723@ntu.edu.cn; dermatochen@163.com; binzane@163.com.
}

Background The present study set out to investigate the epidemiological and clinical characteristics of 65 patients with tsutsugamushi disease.

Methods: The clinical data of 65 patients with tsutsugamushi disease, who were admitted to the Affiliated Hospital of Nantong University were retrospectively analyzed. The clinical symptoms, laboratory examination results, clinical treatment plans, treatments, and outcomes of the patients were analyzed.

Results: The 65 patients with tsutsugamushi disease, included 40 males (61.54\%) and 25 females (38.46\%). The patients were aged from 1 year and 7 months to 88 years old, and the peak age was 60-70 years old. Geographically, the patients were concentrated in Rugao and Tongzhou District. Infections were most common between October and December (categorized as "autumn type"), and peaked in November. Farmers had the highest infection rate of any occupation (66\%). All patients had the symptom of fever, with the body temperature of $60(92.31 \%)$ patients exceeding $38.5{ }^{\circ} \mathrm{C}$, while $58(89 \%)$ and $51(78 \%)$ patients had characteristic eschar and skin rash, respectively. There were $56(86.15 \%)$ patients with varying degrees of liver damage, 8 (12.31\%) cases of elevated D-dimer, 3 (4.62\%) cases of myocardial injury, 38 (58.46\%) cases of superficial lymph node enlargement, 8 (12.31\%) cases of splenomegaly, and 2 cases (3.08\%) of bulbar conjunctival congestion. Of the 65 patients enrolled, the overall misdiagnosis rate of first medical contact was $64.62 \%$

Conclusions: Tsutsugamushi disease, infection has obvious seasonality and a susceptible population, especially among farmers and the elderly.

Koywords! Clinical features; epidemiological features; tsutsugamushi disease; Nantong

Submitted Apr 08, 2021. Accepted for publication May 24, 2021.

doi: 10.21037/apm-21-1100

View this article at: http://dx.doi.org/10.21037/apm-21-1100

\section{Introduction}

Tsutsugamushi disease, also known as scrub typhus, is a zoonosis (1) caused by the gram-negative obligate intracellular pathogen Orientia tsutsugamushi, which is transmitted by arthropods (2). It can cause severe multiple organ failure, and carries a mortality rate of $70 \%$ without appropriate treatment (1). Although tsutsugamushi disease was first reported in Japan in 1899, it did not receive much attention outside Asia until World War II, when a large 
number of soldiers were infected with the disease in the South Pacific region, with the death rate in some places surpassing $30 \%$ (3).

The disease's broad global distribution takes the form of a triangle (1,4-6), within which more than half of the world's population resides (7-9). It threatens 1 billion people globally and causes illness in 1 million people annually. In the Asia Pacific region, tsutsugamushi disease represents a serious public health problem, and one of the main diseases that cause deaths of soldiers in military activities (3), especially in South and East Asia and parts of the Pacific coast, such as China, South Korea, Japan, India, Indonesia, Thailand etc. (2). In Southeast Asia, tsutsugamushi disease is the second largest febrile disease besides malaria (3).

Tsutsugamushi disease has existed in southern China for more than 1,000 years $(7,10)$. In 1948 , the first reported case of scrub typhus was found in Guangzhou, Guangdong $(7,11)$. In 1986, autumn and winter tsutsugamushi disease was identified in Jiangsu province. Since then, an increasing number of people have been infected with this type of tsutsugamushi disease (4). To date, Chinese mainland 31 provinces and cities have recorded local cases (12). In recent years, the incidence rate of tsutsugamushi disease has increased annually. China is one of the main epidemic areas of tsutsugamushi disease, and the disease has become a prominent health problem in the country $(7,9,13)$.

Different geographical, climatic, and environmental factors contribute to different epidemic characteristics of shrub typhus. The peak periods of scrub typhus in mainland China become increasingly longer from the north to the southwest to the south of the country. A single peak exists in southwestern and northern China, but a bimodal peak exists in southern China (14). Typhus is closely related to climate (7), and its prevalence is affected by temperature, rainfall, and other environmental factors $(15,16)$.

Common symptoms of typhus infection include fever, eschar, rash, and elevated liver enzymes $(4,10)$. Without timely treatment, pneumonia, meningitis, myocarditis, or disseminated intravascular coagulation can occur, which may lead to multiple organ failure. In the early stage, tsutsugamushi disease usually manifests as chills, fatigue, and high fever. The symptoms of tsutsugamushi are similar to those of the common cold in the early stage, and the lack of specific main complaints and clinical symptoms means that it may not be noticed by the infected person.

In this research, 65 patients with tsutsugamushi disease who were treated in the Affiliated Hospital of Nantong University between 2015 and 2020 were retrospectively analyzed. The patients' clinical and epidemiological characteristics were summarized, so as to provide awareness programs for clinicians and the public in high-risk areas, and to improve the diagnosis, treatment, and clinical outcomes of this disease. We present the following article in accordance with the STROBE reporting checklist (available at http://dx.doi.org/10.21037/apm-21-1100).

\section{Methods}

\section{Cases}

Sixty-five patients with tsutsugamushi disease were retrospectively enrolled from the Affiliated Hospital of Nantong University from 2015 to 2020 . All cases had completed the report card of infectious diseases of the people's Republic of China. All procedures performed in this study involving human participants were in accordance with the Declaration of Helsinki (as revised in 2013). This study was approved by the ethical committee of the Affiliated Hospital of Nantong University.

\section{Selection criteria}

The diagnosis of tsutsugamushi disease was based on the eighth edition of Infectious Diseases edited by Lanjuan Li. Tsutsugamushi disease was diagnosed if the patient met the following 3 conditions: (I) epidemiological history: a history of fishing, jungle and grassland contact, or field work before onset; (II) clinical manifestations: rash, fever, eschar or ulcer; (III) laboratory tests: Proteus OXK agglutination test agglutination titer $\geq 1: 160$ or positive for Rickettsia tsutsugamushi. Patients who met all the above diagnostic criteria at first onset and provided informed consent to voluntarily participate in the study were included.

\section{Study methods}

In this retrospective study, information including age, sex, occupation, place of residence, and date of disease onset was collected for each of the 65 patients with tsutsugamushi disease, analyzed the percentage of different indicators, and the epidemiological characteristics were summarized. Patients' laboratory data were collected, and the white blood cells (WBC), eosinophil ratio (EO\%), platelet (PLT) count, C-reactive protein (CRP), hematocrit (HCT), platelet distribution width (PDW), albumin (ALB), alanine transaminase (ALT), aspartate transferase (AST), gamma- 


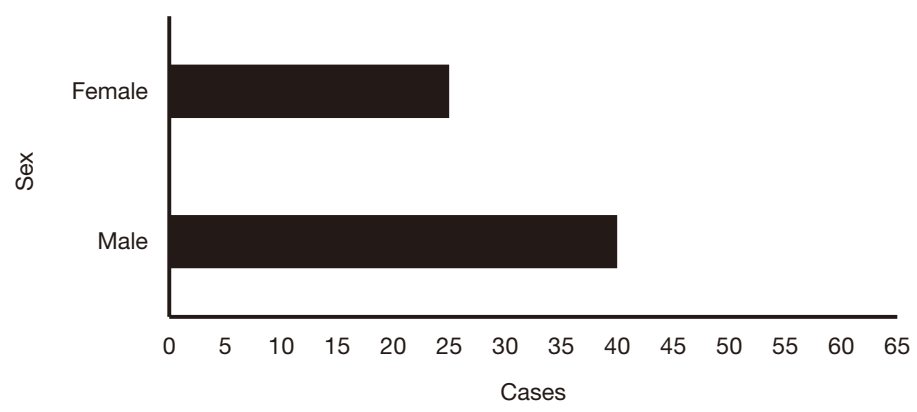

Figure 1 Sex distribution of 65 patients with tsutsugamushi disease.

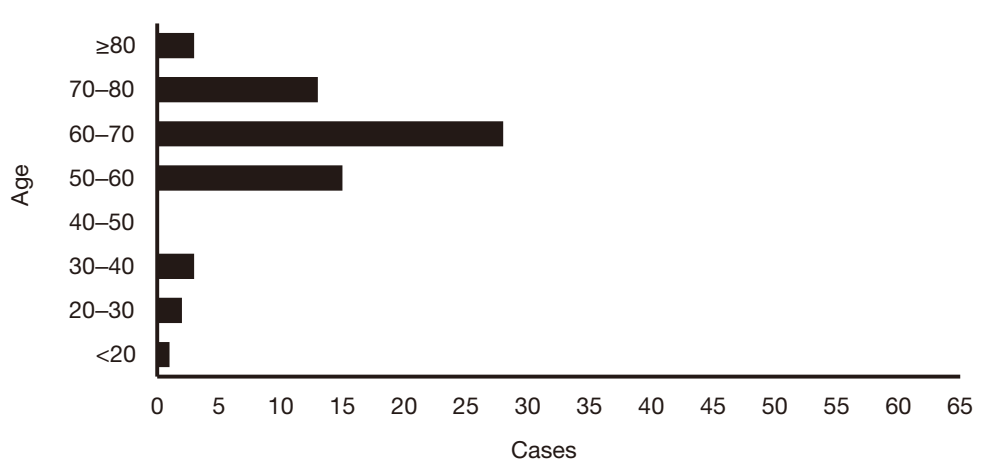

Figure 2 Age distribution of 65 patients with tsutsugamushi disease.

glutamyl transferase (GGT), lactate dehydrogenase (LDH), and creatinine (CR) were compared between two groups(For the 54 patients with liver injury, the median ALT was $96.5 \mathrm{U} / \mathrm{L}$; based on this, the patients were divided into a high expression group (ALT $\geq 96.5 \mathrm{U} / \mathrm{L}$ ) and a low expression group (ALT <96.5 U/L).

\section{Statistical methods}

Continuous variables were expressed as medians (interquartile ranges) or means \pm standard deviations depending on their normality. Categorical variables were expressed as frequencies (percentages). Depending on the distribution of variables, the independent Mann-Whitney U test or Student's $t$-test was used to compare continuous variables between 2 groups. For comparisons between categorical variables, the chi-square test was employed. Fisher's exact test was applied when the expected frequency was $<5$. Spearman's correlation test or Pearson's correlation test was used to assess correlations between 2 continuous variables, as appropriate. Two-tailed $\mathrm{P}<0.05$ was considered statistically significant. Statistical analyses were conducted with GraphPad Prism 8.0 software.

\section{Results}

\section{Epidemiological characteristics}

Age and sex distribution

The 65 study participants included 40 (62\%) males and 25 (38\%) females. The patients had an average age of 68.1 years old (ranging from 1 year and 7 months old to 88 years old). Patients aged 60-70 years had the highest proportion of cases, accounting for $43.08 \%$ of the total cases (Figures 1,2).

\section{Seasonal and regional distribution}

All of the 65 cases of tsutsugamushi disease in this study occurred in October, November, or December, with the majority of cases (53 cases, $81.54 \%$ ) occurring in November. This result suggested that Nantong is an epidemic area of autumn-winter type tsutsugamushi disease (Figure 3). Most of the 65 cases were from Nantong, including Tongzhou District, Rugao City, Rudong County, Qidong City, and Haimen City, but 1 case each came from Shangqiu in Henan province and Dongtai in Jiangsu province. There were $57(87.69 \%)$ and 8 cases $(12.31 \%)$ from rural areas and urban areas, respectively (Figure 4). 


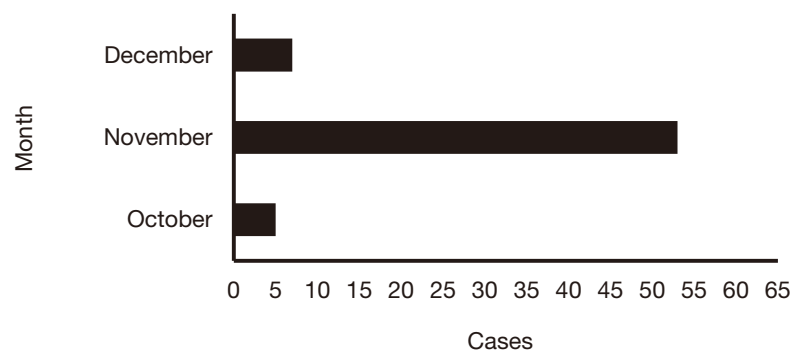

Figure 3 Seasonal distribution of tsutsugamushi disease infections in 65 patients.

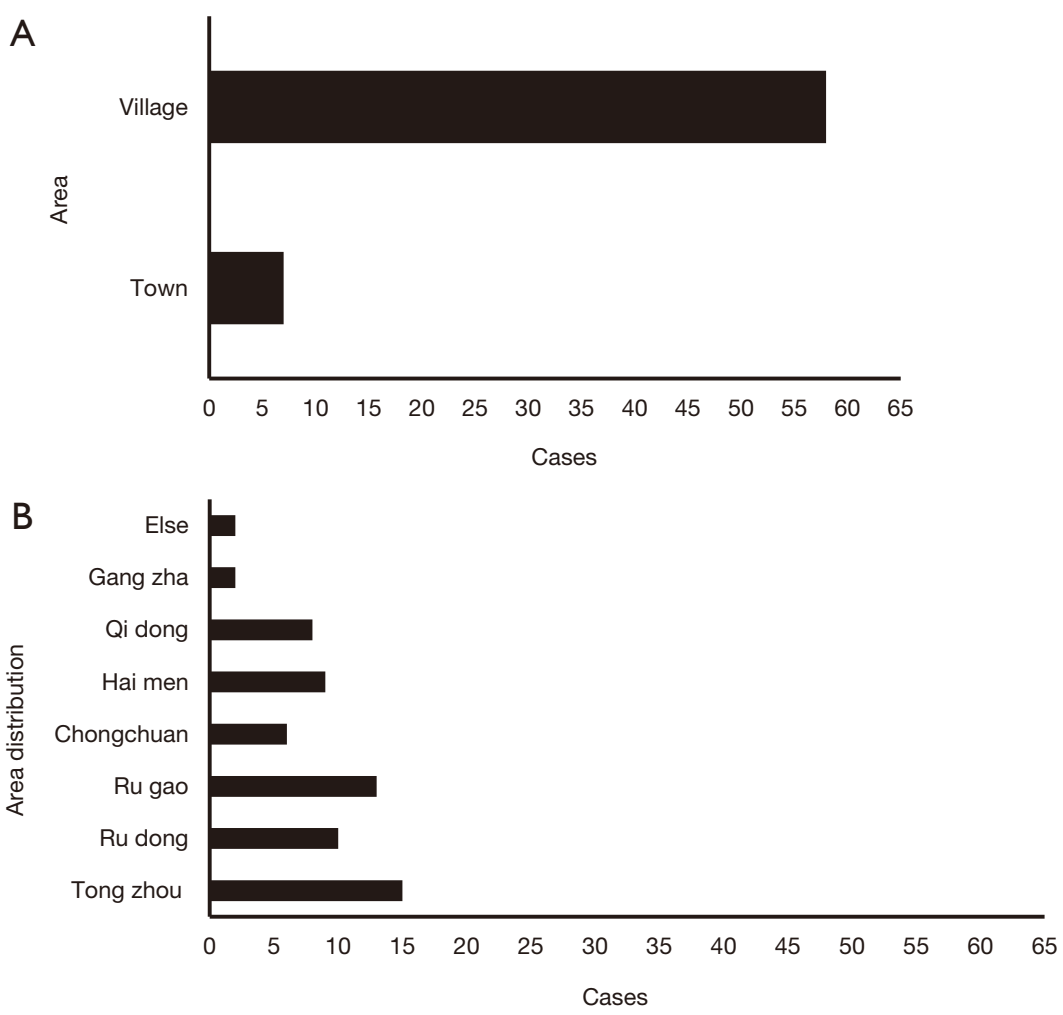

Figure 4 Geographical distribution of 65 patients with tsutsugamushi disease. (A) According to the administrative regions, the number of cases of tsutsugamushi disease. (B) Number of cases of tsutsugamushi disease in various cities of Nantong.

\section{Occupational distribution}

In terms of occupation, farmers accounted for the majority of the 65 enrolled cases (43, 66\%), followed by $11(17 \%)$ retirees, $6(9 \%)$ commercial and service personnel and 5 (8\%) other employees (Figure 5).

\section{Field history}

Of the 65 patients in this analysis, $31(47.69 \%)$ had a history of outdoor activities, 4 (6.15\%) had a history of mosquito bites, and 1 (1.54\%) had a history of aquaculture. The other
29 cases resided in rural areas.

\section{Clinical features}

\section{Fever}

All patients had fever symptoms, including 30 (46.15\%) with moderate fever $\left(38.1-39^{\circ} \mathrm{C}\right)$ and $35(53.85 \%)$ with high fever $\left(39.1-41^{\circ} \mathrm{C}\right)$. Chills, myalgia, fatigue and headache were the most frequent concomitant symptoms (Figures 6,7). 


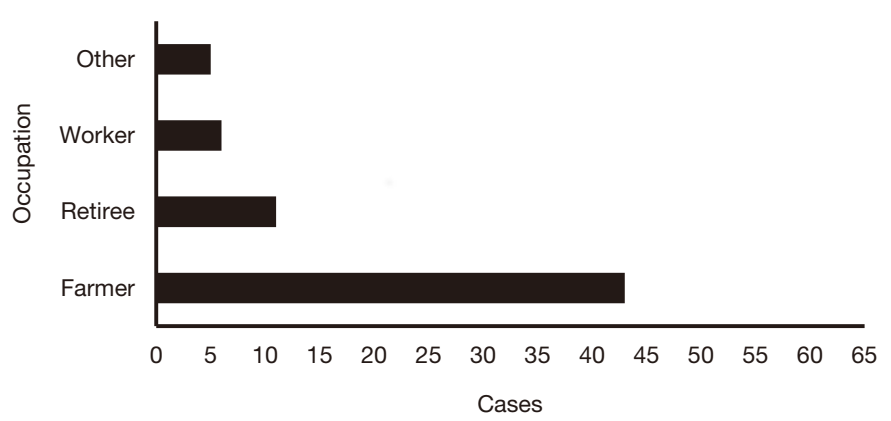

Figure 5 Occupations of 65 patients with tsutsugamushi disease.

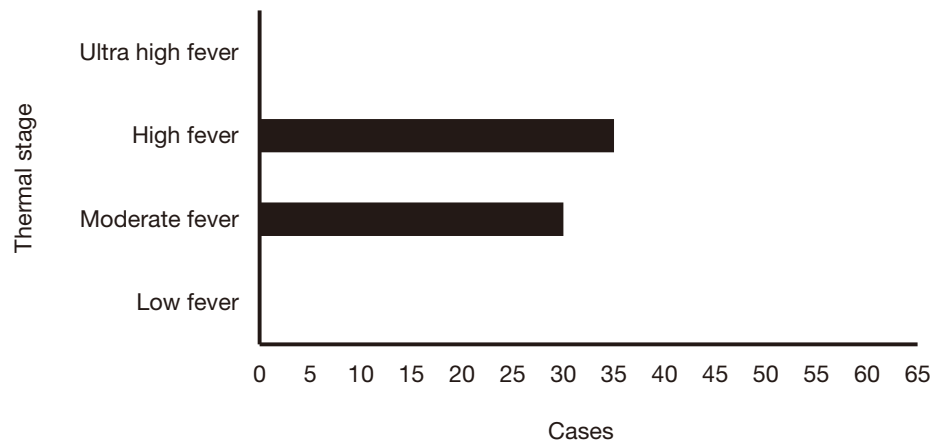

Figure 6 Fever status of patients with tsutsugamushi disease.

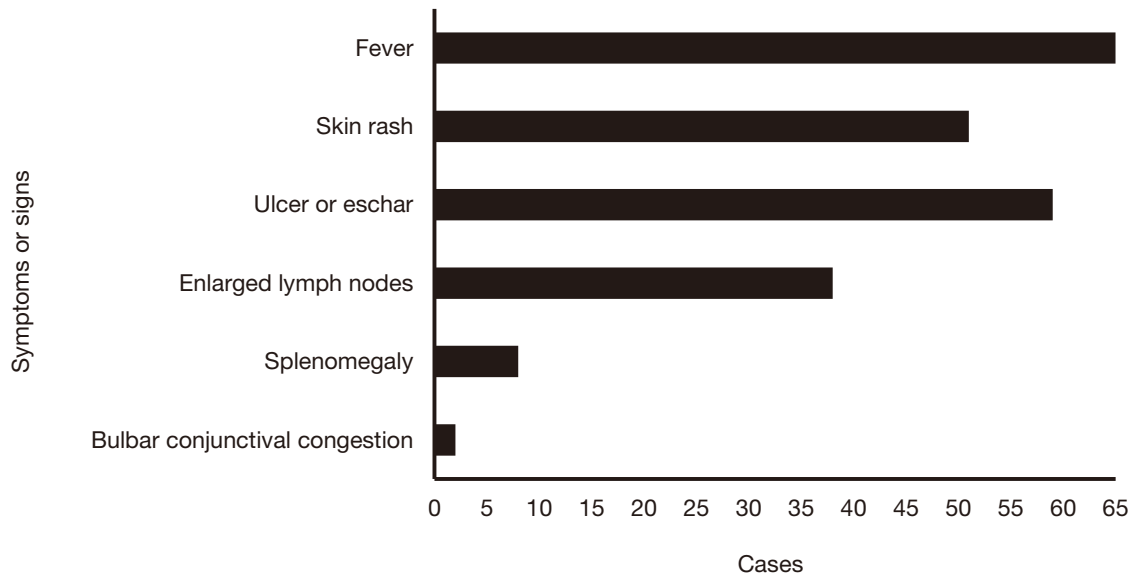

Figure 7 Symptoms or signs of patients with tsutsugamushi disease.

\section{Rash}

There were 51 cases of rash, with commonly affected parts of the body including the face, trunk, chest, and abdomen. In most cases, rash manifested as painless darkred congestive macular papules. The time of eruption was typically about 4 or 5 days after fever (Figure 7).

\section{Eschar and ulcer}

There were $59(90.77 \%)$ cases of eschar or ulcer, including: 10 cases $(16.95 \%)$ in the armpit; 8 cases $(13.56 \%)$ on the ankle; 7 cases $(11.86 \%)$ on the lower limb; 4 cases $(6.78 \%)$ each on the abdomen and breast; 3 cases (5.08\%) each on the neck, clavicle, thigh, and scrotum; 2 cases (3.39\%) each 

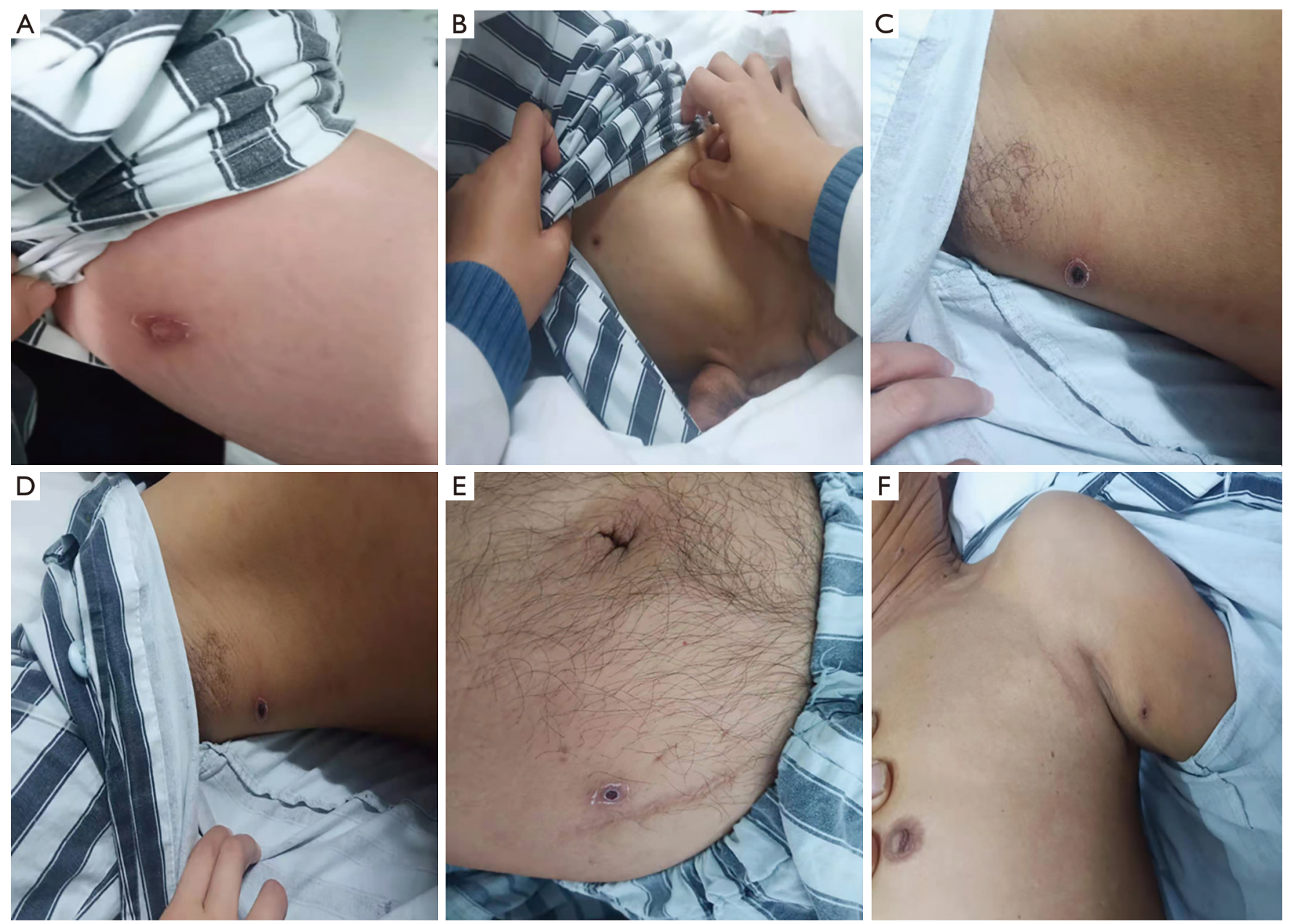

Figure 8 Images of partial eschar in patients with tsutsugamushi disease. (A) The eschar was located on the medial side of the left upper arm, and desquamation appeared in the center of the erythema; (B) the eschar was located on the inner right thigh with red pigmentation; (C) eschar on the right chest wall without painful or itchy; (D) the eschar was located on the right armpit; (E) black crusted lesion on the right lower abdomen with desquamation; (F) the eschar on the inside of the left upper arm fell off to form an ulcer with erythema.

on the waist, shoulder, around the belly button, and at the back knee; and 1 case $(1.70 \%)$ each on the hand, upper limb, and buttock. In all cases, 2 (3.39\%) cases had no less than two eschars or ulcers, and the remaining cases had only one (Figures 7,8).

\section{Lymphadenopathy}

There were 38 (58.46\%) cases of enlarged lymph nodes, which were mainly located in the axilla $(86.84 \%)$, followed by the inguinal region (40\%), neck $(32.31 \%)$, clavicle $(18.46 \%)$, and behind the ear (3.08\%). Twenty-one (32.31\%) patients had enlarged lymph nodes in more than 3 places (Figure 7).

\section{Splenomegaly}

Splenomegaly was found in 8 cases $(12.31 \%)$ by abdominal ultrasound (Figure 7).

\section{Bulbar conjunctiva}

There were $2(3.08 \%)$ cases of bulbar conjunctiva hyperremia (Figure 7).

\section{Clinical laboratory examination}

\section{Blood routine and inflammatory indexes}

Among the 65 patients in the study, 56 patients (86.15\%) had normal leukocytes, 5 cases $(7.69 \%$ ) of leukopenia (with 


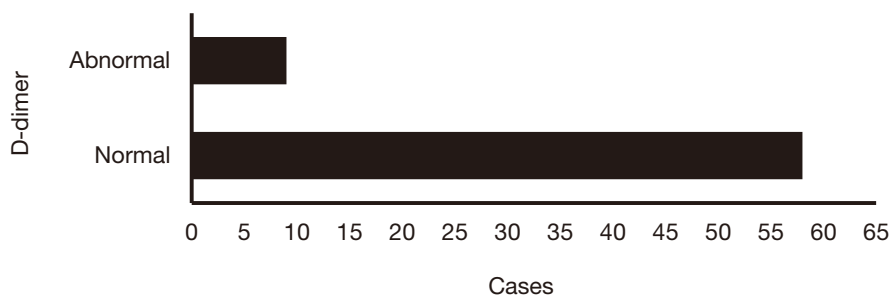

Figure 9 D-dimer levels of patients with tsutsugamushi disease.

the lowest being $\left.2.8 \times 10^{9} / \mathrm{L}\right)$, and 4 patients $(6.15 \%)$ had leukocyte elevation. Almost all of the patients (61 cases, $93.85 \%)$ had a decreased eosinophil percentage. Fifty-two patients $(80 \%)$ had normal platelets, 11 patients $(16.92 \%)$ had thrombocytopenia (with the lowest being $45 \times 10^{9} / \mathrm{L}$ ), and 2 patients $(3.08 \%)$ had elevated platelets (with the highest being $\left.344 \times 10^{9} / \mathrm{L}\right)$. Two cases $(3.08 \%)$ had normal CRP and 63 cases (96.92\%) had elevated CRP.

\section{Biochemical indexes}

Among the 65 cases, liver function was normal in 11 cases (16.92\%) and abnormal in 54 cases $(83.08 \%)$ (AST >40 U/L or ALT $>40 \mathrm{U} / \mathrm{L})$, the renal function was normal in 60 cases $(92.31 \%)$, abnormal in 5 cases $(7.69 \%)$, cardiac markers normal in 62 cases $(95.38 \%$ ) and abnormal in 3 cases $(4.62 \%)$.. These biochemical results were provided by clinical laboratory Affiliated Hospital of Nantong University.

\section{Blood coagulation}

Nine (13.85\%) patients had D-dimer elevation (Figure 9).

\section{Laboratory examination results}

For the 54 patients with liver injury, the median ALT was $96.5 \mathrm{U} / \mathrm{L}$; based on this, the patients were divided into a high expression group (ALT $\geq 96.5 \mathrm{U} / \mathrm{L}$ ) and a low expression group (ALT <96.5 U/L) (Figure 10, Table 1). No significant difference was found between the high and low expression groups in terms of WBC, PLT count, HCT, PDW, EO\%, CRP levels, ALB levels, or CR levels. However, significant differences were found in the levels of AST $(193.9 \pm 133.6$ vs. $80.00 \pm 30.23 ; \mathrm{P}<0.0001)$, $\operatorname{ALP}(182.5 \pm 124.5$ vs. 95.93 $\pm 39.63 ; \mathrm{P}=0.0011)$, GGT, $(182.5 \pm 124.5$ vs. $95.93 \pm 39.63 ; \mathrm{P}=0.0011)$, and $\mathrm{LDH}$ $(964.3 \pm 604.3$ vs. $646.5 \pm 312.6 ; \mathrm{P}=0.0187)$ between the 2 groups.

\section{Relationship of LDH with ALT, AST, and PLT in tsutsugamushi disease (Figure 11)}

LDH was positively correlated with AST $(\mathrm{P}<0.01, \mathrm{r}=0.4639$; Figure $11 \mathrm{~A})$ and $\mathrm{ALT}(\mathrm{P}<0.01, \mathrm{r}=0.3744$; Figure $11 \mathrm{C})$, but negatively correlated with PLT $(\mathrm{P}<0.01, \mathrm{r}=-0.4607$; Figure 11B).

\section{Treatments and outcomes}

Among the 65 patients, 49 patients were treated with minocycline alone, 7 patients were treated with minocycline and levofloxacin, 4 patients were treated with minocycline and piperacillin, 2 patients were treated with minocycline and ganciclovir, 1 patient was treated with minocycline and amoxicillin, 1 patient was treated with azithromycin due to severe liver damage, and 1 patient was treated with cefminocycline and erythromycin. The body temperature of all patients dropped to a normal level within 72 hours after taking the medicine. All patients were cured and discharged from the hospital, and no serious complications or deaths occurred.

\section{Discussion and conclusion}

The clinical symptoms of tsutsugamushi disease are sudden onset, high fever, and characteristic eschar or ulcer of the skin. The disease mainly involves the lung, liver, kidney, spleen, and central nervous system, and its target cells are vascular endothelial cells and macrophages. It can spread to multiple organs through blood flow and lymphocytes. Rickettsia tsutsugamushi is mainly localized in the macrophages of the liver and spleen, which can release toxins to cause hepatocyte inflammation, degeneration, inflammation of hepatic small vessels and liver sinuses, resulting in hepatocyte membrane rupture and microcirculation disturbance. Central liver necrosis, interstitial hepatitis, sinusoid hemorrhage, and hepatocyte 
A

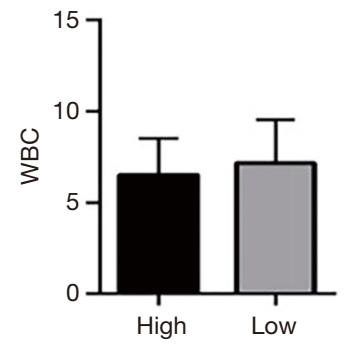

D

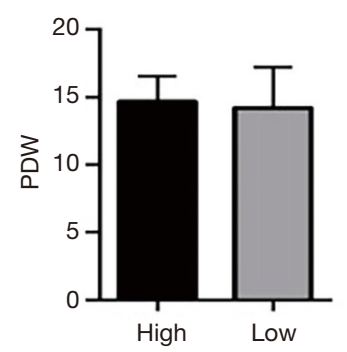

G

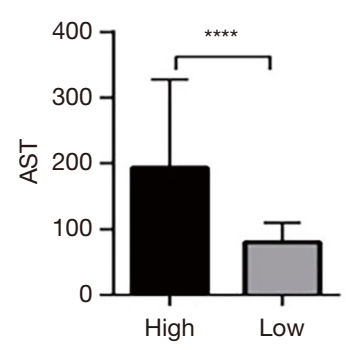

J

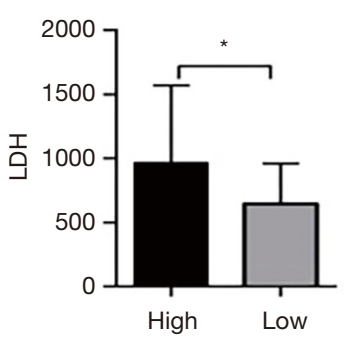

B

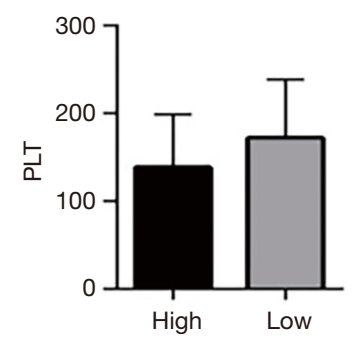

E

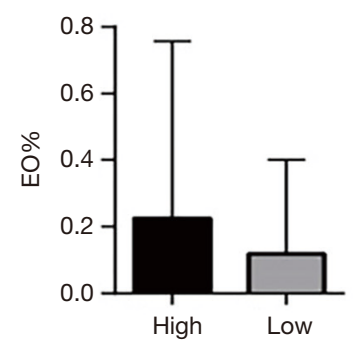

$\mathrm{H}$

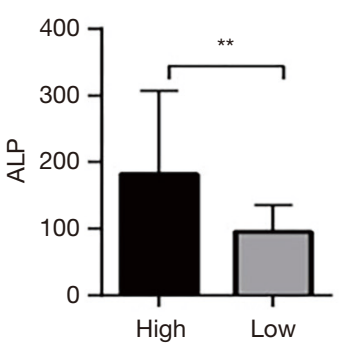

K

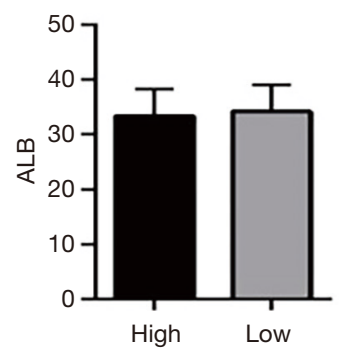

C

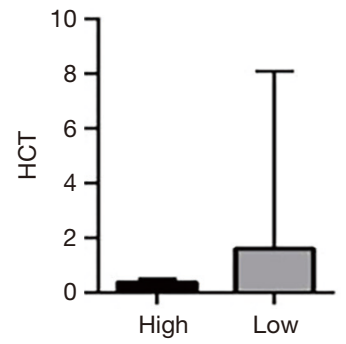

F

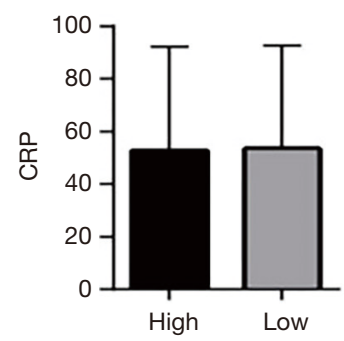

I

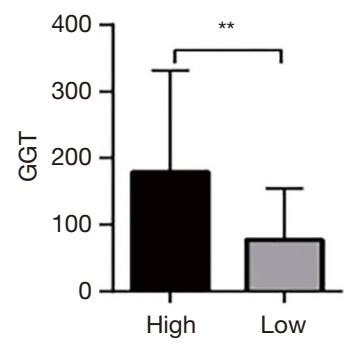

$\mathrm{L}$

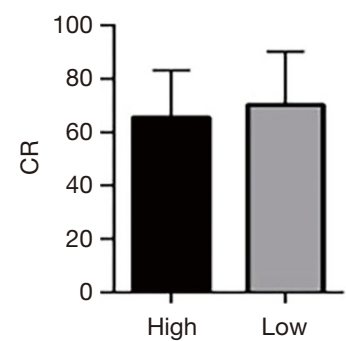

Figure 10 Clinical laboratory data of the ALT high expression group and the ALT low expression group. The patients with liver injury were divided into 2 groups according to ALT 96.5 U/L (median): the high expression group (ALT $\geq 96.5 \mathrm{U} / \mathrm{L}$ ), and the low expression group (ALT $<96.5 \mathrm{U} / \mathrm{L})$. (A,B,C,D,E,F,K,L) No significant difference was found between the high and low expression groups in terms of WBC, PLT count, HCT, PDW, EO\%, CRP levels, ALB levels, or CR levels; (G,H,I,J) Significant differences were found in the levels of AST, ALP , GGT, and LDH between the 2 groups. ALT, alanine transaminase; WBC, white blood cells $\left(\times 10^{9} / \mathrm{L}\right)$; PLT, platelet count $\left(\times 10^{9} / \mathrm{L}\right)$; HCT, hematocrit (L/L); PDW, platelet distribution width (\%); EO\%, eosinophil ratio; CRP, C-reactive protein (mg/L); AST, aspartate transferase (U/L); ALP, alkaline phosphatase (U/L); GGT, gamma-glutamyl transferase (U/L); LDH, lactate dehydrogenase (U/L); ALB, albumin (g/L); CR, creatinine ( $\mu \mathrm{mol} / \mathrm{L}) .{ }^{*} \mathrm{P}<0.05 ;{ }^{* *} \mathrm{P}<0.01 ;{ }^{* * * *} \mathrm{P}<0.0001$. 
Table 1 Analysis of laboratory examination results

\begin{tabular}{lccc}
\hline Variable & High expression group (mean \pm SD) & Low expression group (mean \pm SD) & P value \\
\hline WBC & $6.513 \pm 1.995$ & $7.167 \pm 2.359$ & 0.2764 \\
PLT & $138.6 \pm 60.29$ & $172.3 \pm 66.45$ & 0.0566 \\
HCT & $0.3985 \pm 0.1183$ & $1.623 \pm 6.471$ & 0.3301 \\
PDW & $14.67 \pm 1.861$ & $14.21 \pm 3.015$ & 0.5071 \\
EO\% & $0.2264 \pm 0.5304$ & $0.1189 \pm 0.2814$ & 0.3563 \\
CRP & $52.80 \pm 39.35$ & $53.61 \pm 39.02$ & 0.9401 \\
AST & $193.9 \pm 133.6$ & $80.00 \pm 30.23$ & $95.93 \pm 39.63$ \\
ALP & $182.5 \pm 124.5$ & $77.74 \pm 76.67$ \\
GGT & $179.4 \pm 152.0$ & $646.5 \pm 312.6$ & 0.0001 \\
LDH & $964.3 \pm 604.3$ & $34.19 \pm 4.851$ \\
ALB & $33.40 \pm 4.913$ & $70.19 \pm 19.82$ & 0.0031 \\
CR & $65.52 \pm 17.64$ & 0.0187 \\
\hline
\end{tabular}

Data are expressed as mean \pm SD. WBC, white blood cells $\left(\times 10^{9} / \mathrm{L}\right) ; \mathrm{PLT}$, platelet count $\left(\times 10^{9} / \mathrm{L}\right) ; \mathrm{HCT}$, hematocrit $(\mathrm{L} / \mathrm{L}) ; \mathrm{PDW}, \mathrm{platelet}$ distribution width (\%); EO\%, eosinophil ratio; CRP, C-reactive protein (mg/L); AST, aspartate transferase (U/L); ALP, alkalinephosphatase (U/L); GGT, gamma-glutamyl transferase (U/L); LDH, lactate dehydrogenase(U/L); ALB, albumin (g/L); CR, creatinine ( $\mu \mathrm{mol} / \mathrm{L})$.
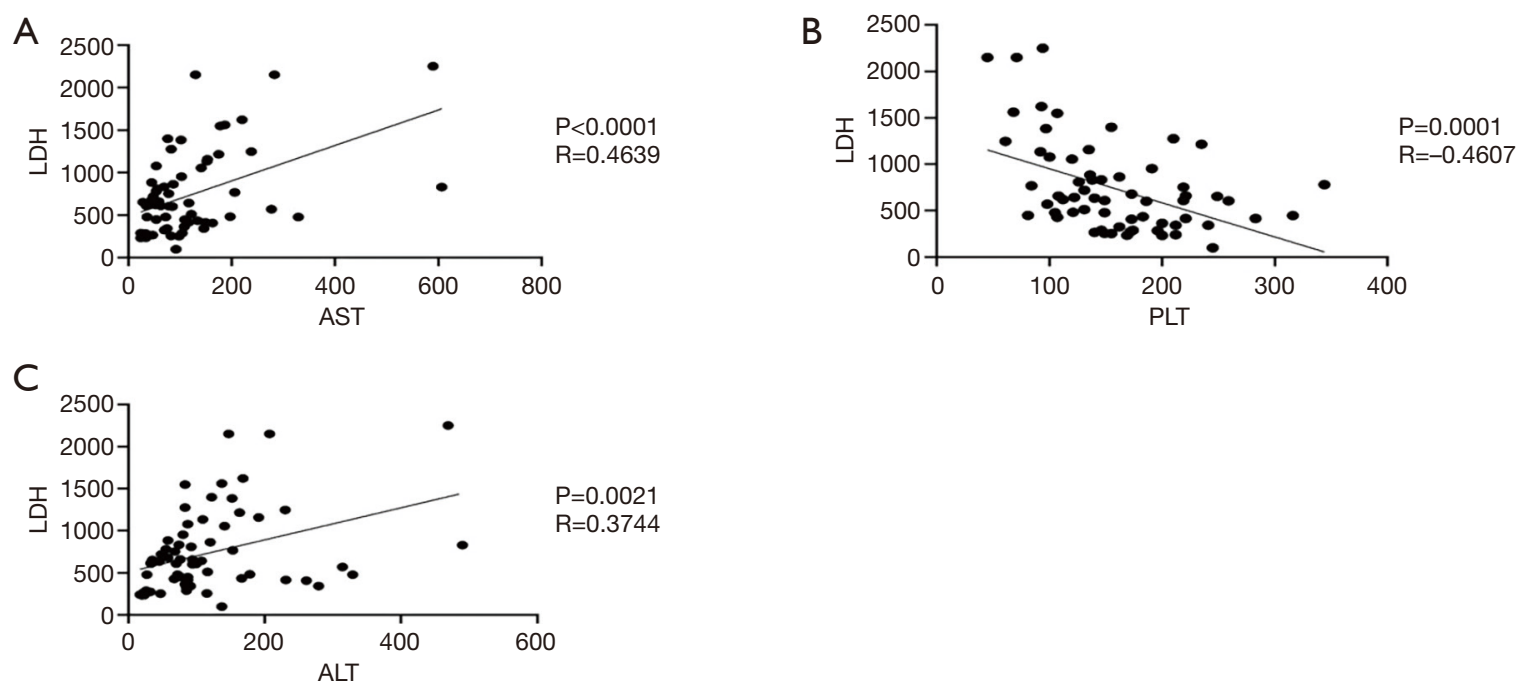

Figure 11 Correlation analysis of LDH, ALT, AST, and PLT in patients with tsutsugamushi disease. (A,C) There was a positive correlation between LDH and AST and ALT in patients with tsutsugamushi disease; (B) LDH and PLT were negatively correlated.

lysis lead to liver injury $(17,18)$. Therefore, the liver is the organ most commonly involved in tsutsugamushi disease, and consequently, liver function injury was taken as the grouping standard in this article.

In this study, the data of 65 patients with tsutsugamushi disease were analyzed retrospectively. Our results showed that all patients experienced fever. Of the 65 patients, $54(83.08 \%)$ had liver injury. ALT, ALP, and LDH were found to be increased. LDH was positively correlated with AST and ALT, but negatively correlated with PLT. This is consistent with the research results of Wang (17) and others. Some studies have evidenced that the decrease 
in the absolute value of EO has good sensitivity and synchronization for tsutsugamushi disease, which is helpful in the diagnosis and curative effect assessment of patients with the disease. Unfortunately, in this study, EO was not compared between tsutsugamushi patients and healthy subjects. Therefore, absolute value of decreasing EO can assist in the diagnosis of fever without typical eschar and epidemiology, and can be used to evaluate the disease severity, and can be used as an early diagnostic index for patients with tsutsugamushi disease.

Laboratory tests for tsutsugamushi disease include serological and molecular biological tests. Crossreactions between Oriental and other rickettsia are rare. The gold standard for the diagnosis of tsutsugamushi disease is indirect immunofluorescence assay. However, unfortunately, this method is expensive and complex, and requires extensive training and the production of reagents for biological containment implementation. Furthermore, the test cannot diagnose infection at an early stage. The molecular biological diagnostic method is to use polymerase chain reaction to detect bacteria, which usually targets the outer membrane gene and GroEL gene of 56 and $47 \mathrm{kDa}$. Nested polymerase chain reaction has been evidenced to potentially be more sensitive than the gold standard indirect immunofluorescence assay (1).

Primary medical institutions are restricted by inspection equipment, inspection technology and other conditions, therefore, the diagnosis of tsutsugamushi disease mainly depends on medical history, signs, and biochemical examinations. All 65 cases in the present investigation had fever, and 59 cases had ulcers or eschar. Among the cases, 42 were misdiagnosed or missed in the first visit, indicating that patients and doctors lack understanding of the physical signs of tsutsugamushi disease, which is related to the fact that eschar or ulcers are often located in concealed parts of the body. The doctor should consider patient's permanent residence and occupation when patient come to us for treatment, and make sure to have a detailed physical examination. To avoid missing important signs, it is also necessary to take into account the local climate, geographical location, and other factors, especially in areas where tsutsugamushi diseases are common.

Nantong is a plain area with a warm and humid climate, which is a suitable environment for rodent growth and reproduction. The main rodents are Rattus norvegicus, Rattus flavipectus, and Rattus norvegicus, which are the main hosts of chigger mites (19). In our study, farmers accounted for $66 \%$ of the patients, and the cases with no clear epidemic history were those living in rural areas, with a large number of cases in Tongzhou District and Rugao. Furthermore, $55.38 \%$ of the patients had a history of outdoor activities, and there was a high incidence of people aged 50 to 80 years old. We also found that the high incidence period was during crop harvesting. These observations fit with the fact that people of an older age make up the main rural labor force, and their work often relates to grasslands, fields, and field work activities. This is consistent with the findings of a previous study (15). Some studies have proved that minocycline is more effective in the treatment of tsutsugamushi disease than other tetracyclines (5), and the therapeutic effect of minocycline has even been used as a diagnostic index (1). Therefore, the active protection of people working outdoors at this time plays an important role in the prevention and treatment of tsutsugamushi disease. Protective measures may include reducing the skin exposure area, using anti-mite sprays, and strengthening rodent control. To summarize, this paper has analyzed the epidemiological characteristics of tsutsugamushi disease. Our findings provide data for the local research of tsutsugamushi disease and will contribute to the prevention and control of tsutsugamushi disease in Nantong. The number of patient samples in this study is too small, which may be explained by the fact that due to the limitation of time and various conditions, this study only collected the clinical data of tsutsugamushi patients who were admitted to the Affiliated Hospital of Nantong University, and did not collect the relevant data of tsutsugamushi patients in other hospitals in the region; because this study is a retrospective study, some Insufficient laboratory examinations have resulted in relatively incomplete experimental data and a small number of standard cases. The true community incidence of the disease may be higher. Therefore, further research is needed to better understand the epidemiology of tsutsugamushi, in order to provide methods for the prevention and treatment of tsutsugamushi.

\section{Acknowledgments}

Funding: This study was supported by the National Natural Science Foundation of China Youth (81801893), the Nantong Clinical Medicine Research Center (HS2019005 and HS2020001), and Nantong Scientific Project (MS12020006 and MS12020017). 


\section{Footnote}

Reporting Checklist: The authors have completed the STROBE reporting checklist. Available at http://dx.doi. org/10.21037/apm-21-1100

Data Sharing Statement: Available at http://dx.doi. org/10.21037/apm-21-1100

Conflicts of Interest: All authors have completed the ICMJE uniform disclosure form (available at http://dx.doi. org/10.21037/apm-21-1100). The authors have no conflicts of interest to declare.

Ethical Statement: The authors are accountable for all aspects of the work in ensuring that questions related to the accuracy or integrity of any part of the work are appropriately investigated and resolved. All procedures performed in this study involving human participants were in accordance with the Declaration of Helsinki (as revised in 2013). This study was approved by the ethical committee of the Affiliated Hospital of Nantong University. Patients who met all the above diagnostic criteria at first onset and provided informed consent to voluntarily participate in the study were included.

Open Access Statement: This is an Open Access article distributed in accordance with the Creative Commons Attribution-NonCommercial-NoDerivs 4.0 International License (CC BY-NC-ND 4.0), which permits the noncommercial replication and distribution of the article with the strict proviso that no changes or edits are made and the original work is properly cited (including links to both the formal publication through the relevant DOI and the license). See: https://creativecommons.org/licenses/by-nc-nd/4.0/.

\section{References}

1. Xu G, Walker DH, Jupiter D, et al. A review of the global epidemiology of scrub typhus. PLoS Negl Trop Dis 2017;11:e0006062.

2. Elliott I, Pearson I, Dahal P, et al. Scrub typhus ecology: a systematic review of Orientia in vectors and hosts. Parasit Vectors 2019;12:513.

3. Kim BN, Gordillo LF, Kim Y. A model for the transmission dynamics of Orientia tsutsugamushi among its natural reservoirs. J Theor Biol 2010;266:154-61.

4. Luo L, Guo Z, Lei Z, et al. Epidemiology of tsutsugamushi disease and its relationship with meteorological factors in Xiamen city, China. PLoS Negl Trop Dis 2020;14:e0008772.

5. Zhao M, Wang T, Yuan X, et al. Comparison of minocycline and azithromycin for the treatment of mild scrub typhus in northern China. Int J Antimicrob Agents 2016;48:317-20.

6. Coeffier M, Gate M, Rimbert A, et al. Validity of Bioimpedance Equations to Evaluate Fat-Free Mass and Muscle Mass in Severely Malnourished Anorectic Patients. J Clin Med 2020;9:3664.

7. Yue Y, Ren D, Liu X, et al. Spatio-temporal patterns of scrub typhus in mainland China, 2006-2017. PLoS Negl Trop Dis 2019;13:e0007916.

8. Seong SY, Choi MS, Kim IS. Orientia tsutsugamushi infection: overview and immune responses. Microbes Infect 2001;3:11-21.

9. Yao H, Wang Y, Mi X, et al. The scrub typhus in mainland China: spatiotemporal expansion and risk prediction underpinned by complex factors. Emerg Microbes Infect 2019;8:909-19.

10. Fan MY, Walker DH, Yu SR, et al. Epidemiology and ecology of rickettsial diseases in the People's Republic of China. Rev Infect Dis 1987;9:823-40.

11. Li T, Yang Z, Dong Z, et al. Meteorological factors and risk of scrub typhus in Guangzhou, southern China, 20062012. BMC Infect Dis 2014;14:139.

12. Wu YC, Qian Q, Magalhaes RJ, et al. Rapid Increase in Scrub Typhus Incidence in Mainland China, 2006-2014. Am J Trop Med Hyg 2016;94:532-6.

13. Wei Y, Huang Y, Li X, et al. Climate variability, animal reservoir and transmission of scrub typhus in Southern China. PLoS Negl Trop Dis 2017;11:e0005447.

14. De W, Jing K, Huan Z, et al. Scrub typhus, a disease with increasing threat in Guangdong, China. PLoS One 2015;10:e0113968.

15. Yu H, Sun C, Liu W, et al. Scrub typhus in Jiangsu Province, China: epidemiologic features and spatial risk analysis. BMC Infect Dis 2018;18:372.

16. Kuo CC, Huang JL, Ko CY, et al. Spatial analysis of scrub typhus infection and its association with environmental and socioeconomic factors in Taiwan. Acta Trop 2011;120:52-8.

17. Wang Y, Li M, Xu W, et al. Analysis of the clinical characteristics of severe tsutsugamushi disease in Yunnan Province from 2017 to 2018. Zhonghua Wei Zhong Bing Ji Jiu Yi Xue 2019;31:1018-23.

18. Lin M, Huang A, Zheng X, et al. Misdiagnosis of scrub 
typhus complicated by hemophagocytic syndrome. BMC Pediatr 2019;19:102.

19. Wu G, Guo H, Yu M. Studies on three types of natural foci of tsutsugamushi disease in eastern part of China.

Cite this article as: $\mathrm{Xu} \mathrm{P}, \mathrm{Mao} \mathrm{G}$, Jiang H, Ren Y, Wang Y, Liang G, Liu W, Zhou Y, Huang Z, Zhang B, Chen X, Qi L. Analysis of the epidemiological and clinical characteristics of 65 patients with scrub typhus on the east coast of China. Ann Palliat Med 2021;10(5):5694-5705. doi: 10.21037/apm-21-1100
Zhonghua Liu Xing Bing Xue Za Zhi 2000;21:34-6.

(English Language Editor: J. Reynolds) 